\title{
Global haemostatic tests in rapid diagnosis and management of COVID-19 associated coagulopathy in acute limb ischaemia
}

\author{
Bingwen Eugene Fan ${ }^{1,2,9,10}$ (1) - Yew Woon Chia 3,9,10 C Christina Lai Lin Sum ${ }^{4}$ Ponnudurai Kuperan 1,2,9,10 . \\ Stephrene Seok Wei Chan ${ }^{1,2,9,10}$. Li Min Ling ${ }^{5,6,9,10}$. Glenn Wei Leong Tan ${ }^{7,9,10}$. Serene Si Ning Goh . Lai Har Wong $^{4}$. \\ Shu Ping Lim ${ }^{4} \cdot$ Kian Guan Eric Lim ${ }^{4} \cdot \mathrm{Hwee} \mathrm{Tat} \mathrm{Tan}^{4} \cdot$ Mui Kia Ang ${ }^{4}$. Soon Lee Lau ${ }^{4}$ Kiat Hoe Ong 1,2,9,10 . \\ Jensen $\mathrm{Ng}^{8,9,10}$
}

Published online: 30 May 2020

(c) Springer Science+Business Media, LLC, part of Springer Nature 2020

To the editor,

COVID-19-associated coagulopathy [1] has recently been described with a globally observed increased incidence of venous thromboembolism (VTE) and arterial thrombosis which manifests as acute myocardial infarction, ischaemic stroke and acute ischaemic limb [2]. The exact pathophysiology of COVID-19 large vessel thrombosis is still under investigation and can be attributed to three plausible causes: firstly, endothelial injury by the SARS-CoV-2 virus that binds via ACE2 receptors on endothelial cells, secondly, a hypercoagulable state from an uncontrolled hyperinflammatory response and thirdly, stasis due to immobilization in critically ill patients. All three causes comprise Virchow's triad of thrombosis.

Bingwen Eugene Fan

Bingwen_Eugene_Fan@ttsh.com.sg

1 Department of Haematology, Tan Tock Seng Hospital, Singapore, Singapore

2 Department of Laboratory Medicine, Khoo Teck Puat Hospital, Singapore, Singapore

3 Department of Cardiology, Tan Tock Seng Hospital, Singapore, Singapore

4 Department of Laboratory Medicine, Tan Tock Seng Hospital, Singapore, Singapore

5 Department of Infectious Diseases, Tan Tock Seng Hospital, Singapore, Singapore

6 National Centre for Infectious Diseases, Singapore, Singapore

7 Department of General Surgery, Tan Tock Seng Hospital, Singapore, Singapore

8 Department of Anaesthesiology, Intensive Care and Pain Medicine, Tan Tock Seng Hospital, Singapore, Singapore

9 Lee Kong Chian School of Medicine, Singapore, Singapore

10 Yong Loo Lin School of Medicine, Singapore, Singapore
We describe a case of a 39-year-old Bangladeshi male migrant worker with no significant past medical history, who presented with fever, cough, and central abdominal discomfort. The patient required supplemental intranasal oxygen of $1.5 \mathrm{l} / \mathrm{min}$ on admission. SARS-CoV-2 PCR from nasopharyngeal swab returned positive and chest X-ray showed bilateral infiltrates. A diagnosis of COVID-19 pneumonia was made, and he was started on intravenous (IV) Remdesivir as part of a clinical trial. He remained ambulant in the general isolation ward and was not commenced on prophylactic anticoagulation upon the managing physician's discretion. On Day 13 of illness, he developed acute right foot pain, with absent distal pedal pulses, decreased warmth, and duskiness of his toes. An acute ischaemic limb was suspected, and urgent vascular surgery consult was obtained.

Global haemostatic tests [3], namely thromboelastography (TEG) (Haemonetics, TEG6s) and clot waveform analysis (CWA) on Sysmex CN-6000 automated coagulation analyser (Sysmex Corporation, Kobe, Japan) with Dade Actin FSL (Siemens Healthcare, Marburg, Germany) were performed within an hour of presentation. These tests helped in the rapid confirmation of the clinical suspicion of a hypercoagulable state in this patient. An urgently arranged CT aortogram later revealed extensive arterial thrombosis presenting as a $2 \mathrm{~cm}$ mobile thoracoabdominal aortic thrombus (Fig. 1), with embolization to the right profunda femoris and popliteal artery. The patient was immediately started on IV unfractionated heparin (UFH) infusion while awaiting further blood investigations and surgery. He underwent a successful endovascular stent graft exclusion of the aortic thrombus and right lower limb embolectomy. Repeat CT aortogram and angiogram on post-operative day 1 revealed no remnant mobile aortic thrombi and successful revascularisation, consistent with restoration of good capillary refill distally and palpable right foot pulses clinically. 


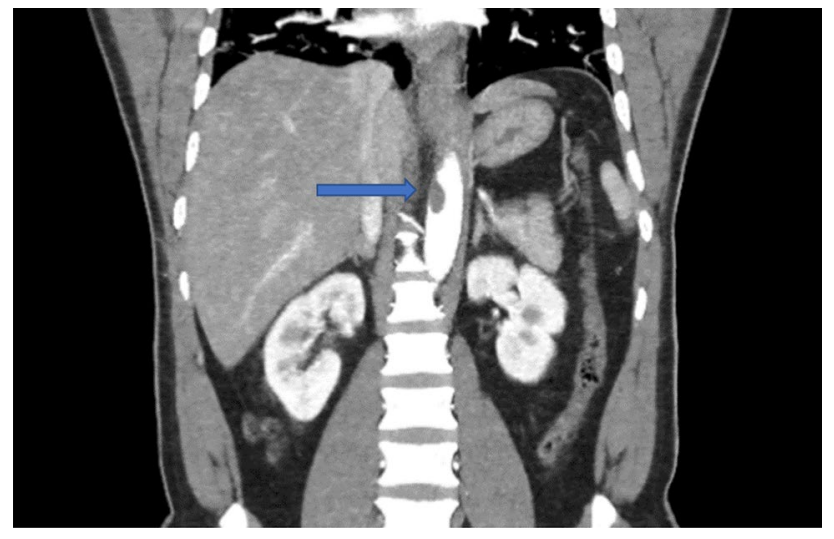

Fig. $12 \mathrm{~cm}$ mobile thoracoabdominal aortic thrombus (blue arrow)

On presentation, TEG (Fig. 2a) showed an increased Citrated RapidTEG (CRT) maximal amplitude (MA) of $71.3 \mathrm{~mm}(52-70 \mathrm{~mm})$ and a Citrated Kaolin (CK) MA of $69.2(52-69 \mathrm{~mm})$, suggestive a hypercoagulable state, and an increased Citrated Functional Fibrinogen (CFF) MA of $43.8 \mathrm{~mm}(15-32 \mathrm{~mm})$ pointing to an excessive fibrinogen component to the clot strength.

CWA performed on activated partial thromboplastin time (aPTT) (Fig. 3) showed markedly raised clot velocity (min 1 of $9.715 \% / \mathrm{s}(2.85-6.65 \% / \mathrm{s}))$, increased clot acceleration ( $\min 2$ of $1.566 \% / \mathrm{s}(0.46-1.08 \% / \mathrm{s}))$, increased clot deceleration $(\max 2$ of $1.311 \% / \mathrm{s}(0.37-0.91 / \mathrm{s}))$, and significant decrease in light transmission (delta change of $0.9644(0.10-0.41)$ ). These parameters were suggestive of a hypercoagulable state.

Standard laboratory investigations performed upon presentation of the acute ischaemic limb also supported diagnosis of a hypercoagulable profile. Both prothrombin time (PT) (14.3 s (11.0-14.0 s)) and aPTT (38.9 s (27.0-37.0 s)) were prolonged, with an uncorrectable APTT on 50:50 mixing study, suggesting the presence of a lupus anticoagulant confirmed by the Dilute Russel Viper venom test (DRVVT). Anti-cardiolipin IgG and IgM were both elevated at 35 GPL units (0-20 GPL) and 28 MPL units (0-20 MPL) respectively. Fibrinogen (7.7 g/L $(1.8-4.5 \mathrm{~g} / \mathrm{L}))$ and D-dimer $(2.55 \mathrm{ug} / \mathrm{ml}(<0.50))$ were elevated, clotting factors were raised, notably Factor II (133\% (70-120\%)), Factor V (134\% (70-120\%)), Factor VIII (498\% (60-150\%)), Factor IX (202\% (60-150\%)) and von Willebrand Antigen was high at $371 \%$ (56-160\%). The patient had normal homocysteine, protein C, protein $\mathrm{S}$ and anti-thrombin levels. His C-reactive protein level was raised at $136.2 \mathrm{mg} / \mathrm{l}(0-5 \mathrm{mg} / \mathrm{L})$. His International Society of Thrombosis and Haemostasis Disseminated Intravascular Coagulopathy (DIC) score was 2 (due to moderate rise of D-dimer), which suggested a low probability of overt DIC.
Fig. 2 a Thromboelastography (TEG) performed at diagnosis of acute ischaemic limb. CK angle $78.6^{\circ}\left(63^{\circ}-78^{\circ}\right)$ and CRT angle $80.3^{\circ}\left(60^{\circ}-78^{\circ}\right)$ are increased, CK MA $69.2 \mathrm{~mm}(52-69 \mathrm{~mm})$ and CRT MA $71.3 \mathrm{~mm}(52-70 \mathrm{~mm})$ are also increased, CFF MA $43.8 \mathrm{~mm}(15-32 \mathrm{~mm})$ is markedly increased secondary to hyperfibrinogenaemia, reflected by high functional fibrinogen (FLEV) $799.3 \mathrm{mg} / \mathrm{dl}$. b Thromboelastography (TEG) on post-operative day 1 performed on citrated blood sample with IV heparin infusion. CK $\mathrm{R}$ is markedly prolonged $20.7 \mathrm{~min}$ (4.6-9.1 $\mathrm{min}$ ) due to the heparin effect, while CKH R is borderline prolonged $10.5 \mathrm{~min}$ (4.3-8.3 min). CKH MA is still raised at $69.3 \mathrm{~mm}(52-69 \mathrm{~mm})$, suggestive of a persistent hypercoagulable state in the absence of heparin, while CK MA is normal, suggestive that clot formation is normalised in presence of heparin. CFF MA $53.8 \mathrm{~mm}(15-32 \mathrm{~mm})$ is further increased secondary to hyperfibrinogenaemia, reflected by higher functional fibrinogen (FLEV) $981.8 \mathrm{mg} / \mathrm{dl}$ which may be reflective of the postoperative infective systemic inflammatory milieu

Pre-operatively, IV UFH was titrated to $1300 \mathrm{U} / \mathrm{h}$ to achieve an anti-Xa level of $0.4-0.6 \mathrm{IU} / \mathrm{ml}$ then discontinued for surgery. Post-operatively, IV UFH infusion without a bolus dose was resumed but anti-Xa levels rose gradually but remained persistently below $0.2 \mathrm{IU} / \mathrm{ml}$, which was slightly under our intended therapeutic target of $0.3-0.7 \mathrm{IU} /$ $\mathrm{ml}$. This was likely due to the post-operative inflammatory milieu causing a rise in acute phase proteins that bind to heparin. Heparin resistance was unlikely as anti-thrombin III levels were rechecked and were normal at $94 \%$. On the repeat TEG (Fig. 1b) the CKH-R was prolonged relative to CK-R (20.7 vs $10.5 \mathrm{~min}$ ), demonstrating UFH effect, as well as a mildly increased CKH MA of $69.3 \mathrm{~mm}(52-69 \mathrm{~mm})$, pointing to a persistent hypercoagulable state. IV UFH was later switched to low molecular weight heparin (LMWH) on post-operation day 1 . While on LMWH, the patient was subsequently able to attain a mean anti-Xa level of $0.61 \mathrm{IU} /$ $\mathrm{ml}$. Aspirin was started as an antiplatelet agent to prevent in-stent thrombosis.

Lupus anticoagulant associated with COVID-19-infection has been described [4] together with antiphospholipid antibodies that occur with thrombotic events [5], with false positive rates described in aPTT based assays for lupus anticoagulant in the presence of raised CRP which can bind to phospholipids [6]. In our centre, our lupus anticoagulant test is based on DRVVT which is less likely affected by the raised CRP. The mildly raised anti-cardiolipin antibodies of IgG 35 GPL and IgM 28 MPL did not meet the required laboratory criteria (revised Sapporo criteria) of a moderate to high titre of $>40 \mathrm{GPL}$ units for anticardiolipin IgG and $>40$ MPL for anticardiolipin IgM, for the diagnosis of antiphospholipid syndrome (APS).

It is still unclear if the presence of antiphospholipid antibodies in COVID-19 infection is part of an epiphenomenon or is actually involved as a contributory cause to thrombotic events in patients with COVID-19 associated coagulopathy [7]. We arrived at the possible diagnosis of a COVID-19 acquired APS given the presence of a lupus anticoagulant 


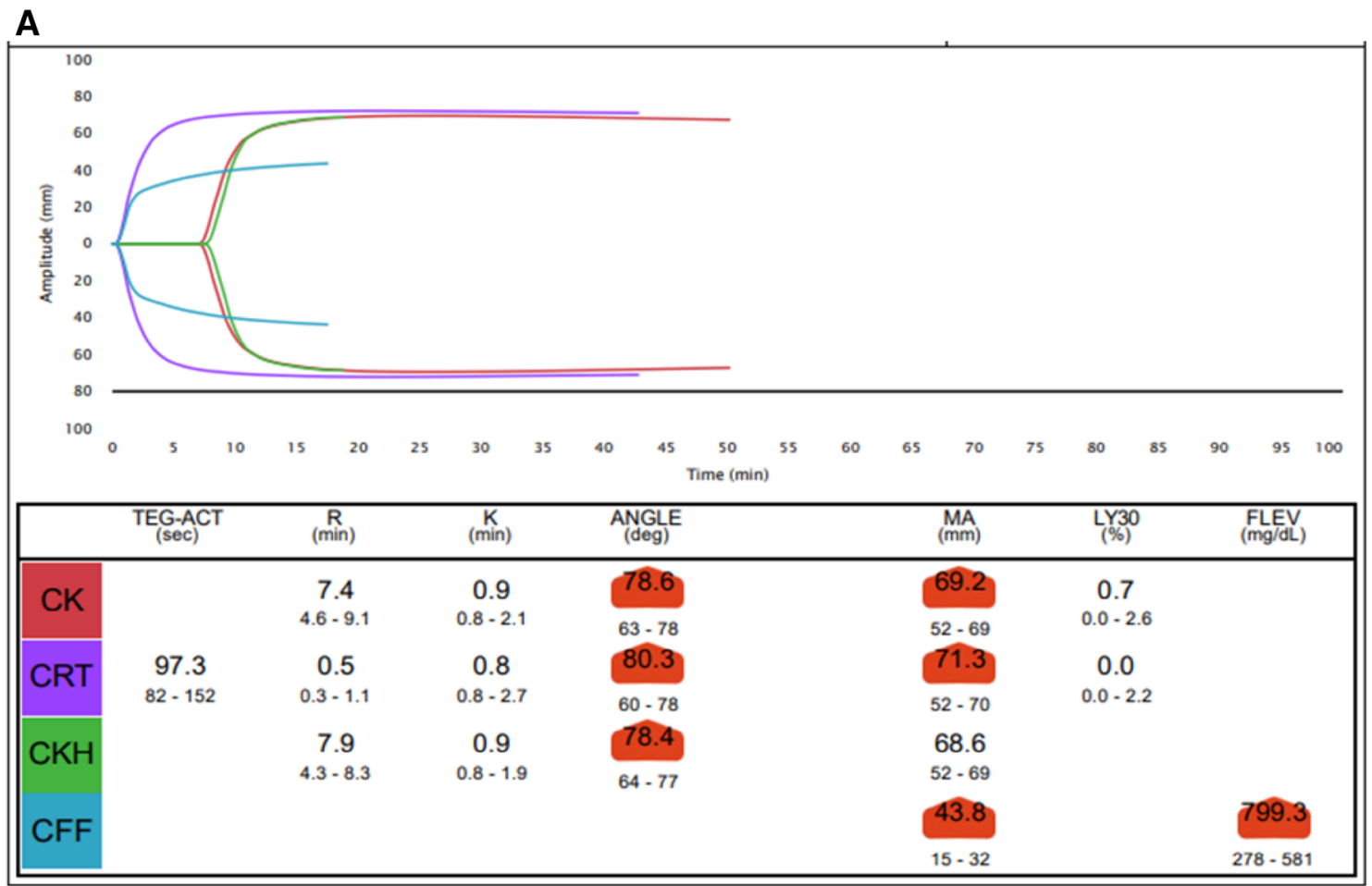

B

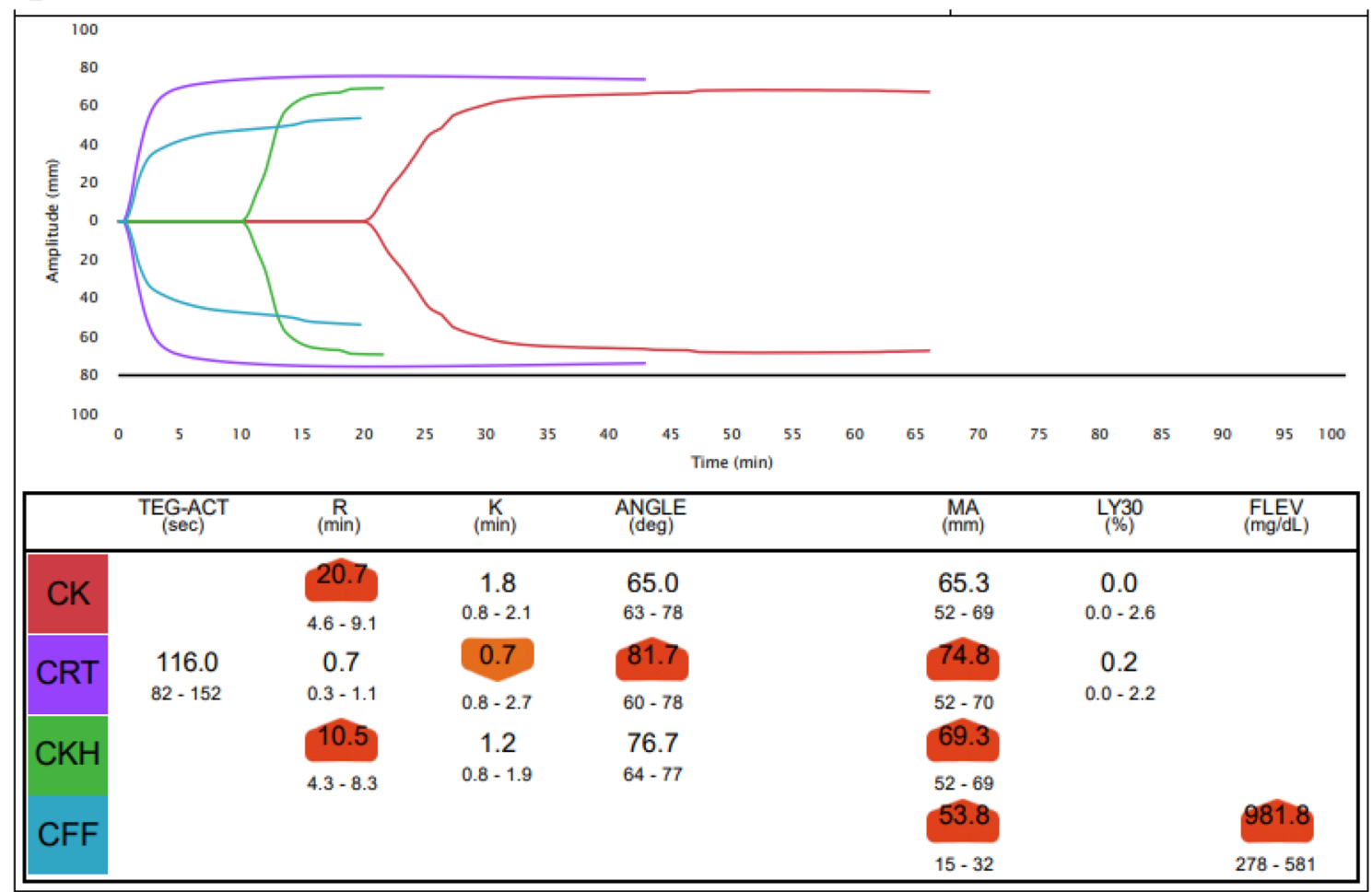




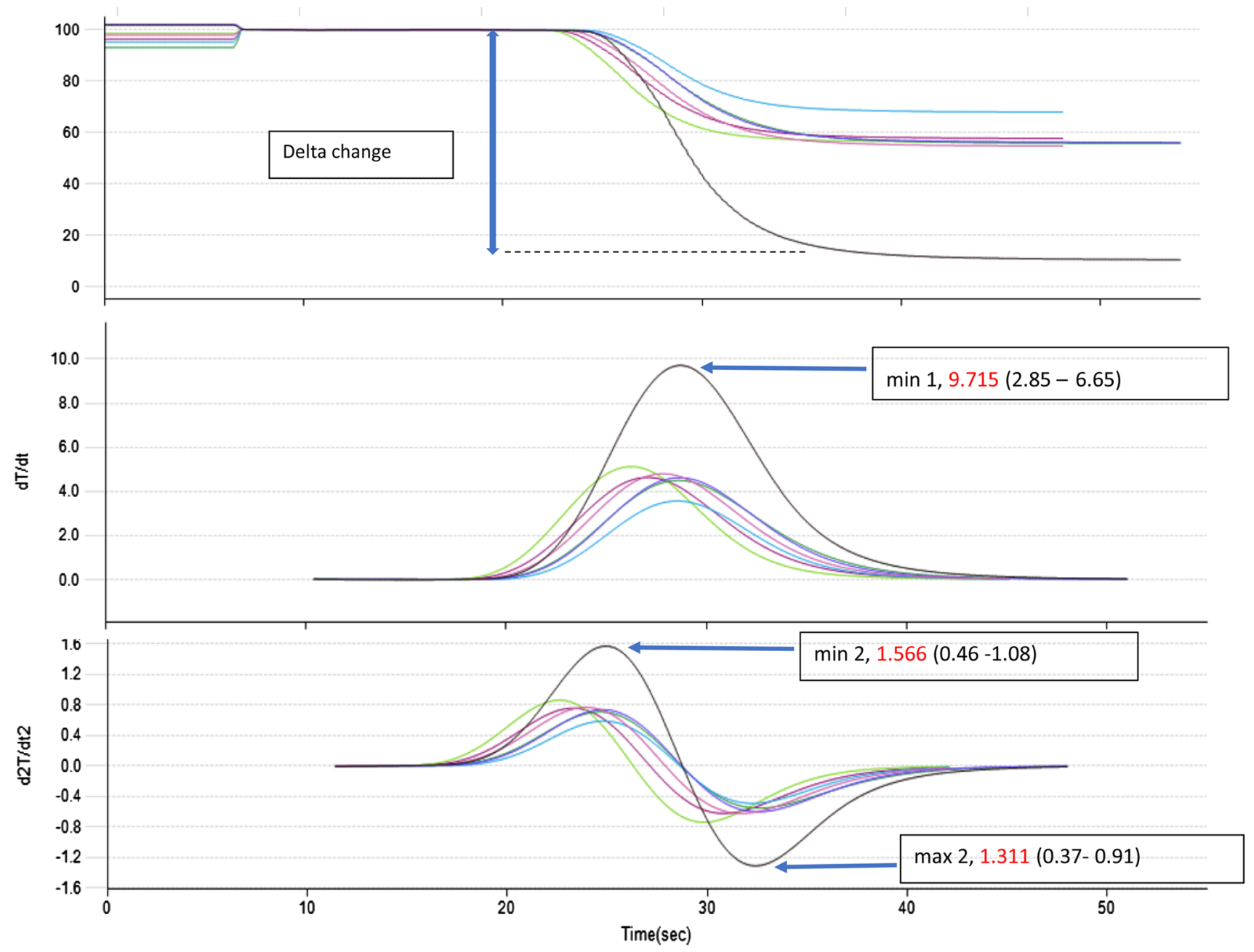

Fig. 3 aPTT clot waveform analysis showing markedly raised min1 of $9.715 \% / \mathrm{s}, \min 2$ of $1.566 \% / \mathrm{s}, \max 2$ of $1.311 \% / \mathrm{s}$ and delta change of 0.9644 , suggestive of a hypercoagulable state (patient's clot waveform curve in black in comparison with 5 normal healthy individuals multicolour clot waveform curves). Reference ranges for min 1 (2.85-

and presentation of acute, extensive arterial thrombosis in an otherwise healthy, young male with severe COVID-19 infection. Presence of pre-existing antiphospholipid antibodies in this patient was a possibility too, with antiphospholipid antibodies resulting in a higher baseline risk of thrombosis, that was exacerbated by the prothrombotic state due to the severe COVID-19 infection, causing arterial thrombosis. Hence, the decision was made to continue anticoagulation with warfarin for at least 12 weeks before repeating the antiphospholipid screen, to confirm our suspicion of an acquired antiphospholipid syndrome.

We wish to share 5 points in utilizing global haemostatic tests to aid in diagnosis of COVID-associated coagulopathy and in the management of anticoagulation in COVID-19-associated acute limb ischaemia.
$6.65 \% / \mathrm{s}), \min 2(0.46-1.08 \% / \mathrm{s}), \max 2(0.37-0.91 \% / \mathrm{s})$, delta change $(0.10-0.41)$ were established at our centre based on 105 healthy individuals in accordance with the Clinical and Laboratory Standards Institute guidelines

(1) TEG and CWA appear sensitive in detecting hypercoagulable states in patients with COVID-19 associated coagulopathy.

Use of TEG and ROTEM in detection of hypercoagulable states have previously been described in malignancy associated coagulopathy and thrombosis, as well as in postoperative hepatic, intrabdominal surgery and neurosurgery. In addition, TEG has shown hypercoagulability in patients with coronary artery disease, oral contraceptives, burn patients and in major orthopaedic trauma injuries [8, 9]. A hypercoagulable state in ICU patients with COVID-19 infection has been reported by Panigada et al [10], where TEG parameters were consistent with hypercoagulability as demonstrated by decreased reaction time ( $\mathrm{R}$ value) and speed of clot formation ( $\mathrm{K}$ value), and increased values of alpha angle 
and MA. While no arterial thrombosis was reported in that study, the TEG findings support the associated events of VTE mentioned.

CWA evaluates clot formation kinetics within the standard PT and aPTT clotting tests, with higher CWA parameters associated with high Padua prediction score for venous thromboembolism, suggesting this as a useful parameter for hypercoagulability [11]. Elevated CWA parameters have also been shown to be associated with VTE [12]. Tan et al. reported in ICU patients with COVID-19 infection [13], a higher min1 value with less elevated $\min 2$ and $\max 2$ levels, reflective of a hypercoagulable state in COVID-19 infection, and suggested a positive association between the elevation of CWA parameters with worsening severity of COVID19 infection.

(2) TEG appears a useful tool in detecting hypercoagulability even in the presence of heparin.

In our patient, heparin effect was detected on TEG even though measured anti-Xa activity was sub-therapeutic. The failure to attain target anti-Xa levels after restarting IV UFH post-operatively was likely due to the inflammatory milieu causing a rise in acute phase proteins that bind to heparin.

TEG has heparinase in the Citrated Kaolin with Heparinase $(\mathrm{CKH})$ channel which neutralises the effect of heparin in whole blood. A repeat TEG (Fig. 2b) was performed to look for heparin effect which was present as CK-R was markedly prolonged relative to CKH-R. While the effect of heparin on global haemostasis can be observed using TEG but cannot be accurately quantified, TEG is a useful tool in detecting hypercoagulability even in the presence of heparin by demonstrating differences between the MA parameter in the CK and CK-H channels.

(3) Use of global haemostatic tests (TEG and CWA) in the setting of an antiphospholipid syndrome.

TEG has been used to assess haemostasis in patients with APS while on long term anticoagulation and/or antiplatelets, with results from the TEG not showing a major bleeding propensity [14]. It has also been used in comparing individuals with antiphospholipid antibodies with patients with primary APS, with healthy individuals with antiphospholipid antibodies and patients with primary APS both not demonstrating a hypercoagulable state by TEG criteria [15]. These two studies show that the presence of antiphospholipids or antiphospholipid syndrome alone does not result in TEG reflecting either a major bleeding profile or hypercoagulable state. Hence, the findings of a hypercoagulable state in our patient on TEG (Fig. 2a, b) may be attributed to COVID-19 associated coagulopathy. No formal studies on CWA assessing clotting parameters in APS have been described.

(4) Rapid turnaround time of global haemostatic tests (TEG and CWA).

While not clinically accepted as standard point of care tests for assessment of hypercoagulable states, given the rapid turnaround time (usually within $1 \mathrm{~h}$ ) compared to conventional tests of haemostasis, TEG and CWA may be useful adjunctive tools to help clinicians rapidly assess the haemostatic profile of the patient in time-sensitive conditions such as an acute ischaemic limb.

(5) Infection control considerations.

Many of the novel research haemostatic tests cannot be safely performed on blood specimens of COVID19 patients given laboratory biosafety concerns, due to risk of aerosolization. Newer models of viscoelastic tests such as TEG6s and ROTEM-Sigma are small enough to be placed in a Class II biosafety cabinet. This allows flexibility of having these as a point of care test in the ICU or operation theatre setting during the COVID-19 pandemic.

In summary, the results observed in our patient with an acute ischaemic limb support a hypercoagulable state seen in COVID-19 associated coagulopathy, with a probable diagnosis of antiphospholipid syndrome. Global haemostatic tests, while considered mainly as haemostasis research parameters, may be useful in the early identification of a hypercoagulable state and management of thrombotic scenarios in COVID-19 infection. More studies and clinical trials are awaited to evaluate the utility of such tests to support the diagnosis of COVID-19 associated coagulopathy.

Acknowledgements The COVID-19 Clotting and Bleeding Team at the National Centre for Infectious Diseases, Singapore greatly appreciates the efforts of our fellow healthcare workers and the support of their families during this pandemic. Special thanks to Transmedic Pte., Ltd. (Singapore) for their technical support and loan of TEG6, and Sysmex Corporation (Japan) for their technical support and loan of CN-6000.

\section{Compliance with ethical standards}

Conflict of interest The authors declare that they have no conflict of interest.

Ethics approval This study was approved by the National Healthcare Group Domain Specific Review Board (DSRB). Informed consent was obtained from the patient. 


\section{References:s}

1. Becker RC (2020) COVID-19 update: Covid-19-associated coagulopathy. J Thromb Thrombolysis. https://doi.org/10.1007/s1123 9-020-02134-3

2. Perini P, Nabulsi B, Massoni CB, Azzarone M, Freyrie A (2020) Acute limb ischaemia in two young, non-atherosclerotic patients with COVID-19. Lancet. https://doi.org/10.1016/S0140 $-6736(20) 31051-5$

3. Lancé MD (2015) A general review of major global coagulation assays: thrombelastography, thrombin generation test and clot waveform analysis. Thromb J 13:1. https://doi. org/10.1186/1477-9560-13-1

4. Harzallah I, Debliquis A, Drénou B (2020) Lupus anticoagulant is frequent in patients with Covid-19. J Thromb Haemost. https:// doi.org/10.1111/jth.14867

5. Zhang Y, Xiao M, Zhang S, Xia P, Cao W, Jiang W et al (2020) Coagulopathy and antiphospholipid antibodies in patients with Covid-19. N Engl J Med. https://doi.org/10.1056/NEJMc2007575

6. Schouwers SM, Delanghe JR, Devreese KM (2010) Lupus anticoagulant (LAC) testing in patients with inflammatory status: does C-reactive protein interfere with LAC test results? Thromb Res 125(1):102-104. https://doi.org/10.1016/j.thromres.2009.09.001

7. Connell NT, Battinelli EM, Connors JM (2020) Coagulopathy of COVID-19 and antiphospholipid antibodies. J Thromb Haemost. https://doi.org/10.1111/jth.14893

8. Walsh M, Moore EE, Moore H, Thomas S, Lune SV, Zimmer D et al (2019) Use of viscoelastography in malignancy-associated coagulopathy and thrombosis: a review. Semin Thromb Hemost 45(04):354-372. https://doi.org/10.1055/s-0039-1688497

9. Toukh M, Siemens DR, Black A, Robb S, Leveridge M, Graham CH, Othman M (2014) Thromboelastography identifies hypercoagulablilty and predicts thromboembolic complications in patients with prostate cancer. Thromb Res 133(1):88-95

10. Panigada M, Bottino N, Tagliabue P et al (2020) Hypercoagulability of COVID-19 patients in intensive care unit. A report of thromboelastography findings and other parameters of hemostasis. J Thromb Haemost. https://doi.org/10.1111/jth.14850

11. Ruberto MF, Marongiu F, Mandas A, Mameli A, Porru M, Cianchetti $\mathrm{E}$ et al (2018) The venous thromboembolic risk and the clot wave analysis: a useful relationship? Clin Chem Lab Med 56:448-453. https://doi.org/10.1515/cclm-2017-0678

12. Tan CW, Cheen MHH, Wong WH et al (2019) Elevated activated partial thromboplastin time-based clot waveform analysis markers have strong positive association with acute venous thromboembolism. Biochem Med 29(2):020710

13. Tan CW, Low JGH, Wong WH, Chua YY, Goh SL, Ng HJ (2020) Critically ill COVID-19 infected patients exhibit increased clot waveform analysis parameters consistent with hypercoagulability. Am J Hematol. https://doi.org/10.1002/ajh.25822

14. Wallace NH, Dumont A, Burns A, Christopher TA, Rinder HM, Kriegel MA, Buckley LM, Erkan D, Lee AI (2016) Thromboelastography in the characterization of coagulation status in antiphospholipid syndrome. Blood 128(22):4983. https://doi.org/10.1182/ blood.V128.22.4983.4983

15. Karen AB, Beverley JH (2010) The utility of thromboelastography (TEG) in the management of patients with isolated antiphospholipid antibodies (aPL) or primary antiphospholipid syndrome (PAPS). Blood 116(21):3179. https://doi.org/10.1182/blood . $\mathrm{V} 116.21 .3179 .3179$

Publisher's Note Springer Nature remains neutral with regard to jurisdictional claims in published maps and institutional affiliations. 